\title{
MODELAGEM DE MORTALIDADE EM FLORESTAS NATURAIS
}

\author{
Luiz Marcelo Brum Rossi*, Henrique Soares Koehler**, Carlos Roberto Sanquetta***, \\ Julio Eduardo Arce*** \\ *Eng. Florestal, M.Sc., Embrapa Amazônia Ocidental - mrossi@cpaa.embrapa.br \\ **Eng. Florestal, Dr., Depto. de Fitotecnia e Fitossanitarismo, UFPR - koehler@ufpr.br \\ ***Eng. Florestal, Dr., Depto. de Ciências Florestais, UFPR - sanquetta@ufpr.br - jarce@ufpr.br \\ Recebido para publicação: 28/08/2006 - Aceito para publicação: 23/11/2006
}

\begin{abstract}
Resumo
As florestas naturais são ecossistemas complexos para os quais freqüentemente não existem dados e métodos adequados para a descrição do comportamento de modelos de crescimento e produção. $\mathrm{O}$ recrutamento, o crescimento e a mortalidade são os três componentes básicos para a modelagem de uma floresta. Neste trabalho, o objetivo foi fornecer uma visão geral da modelagem da mortalidade aplicada em florestas naturais. A revisão apresenta aspectos gerais da ocorrência, causas e tipos de mortalidade que ocorrem em florestas; a obtenção de dados e estimativa da mortalidade a partir de inventários; os valores das taxas de mortalidade em diferentes florestas naturais; as maneiras de abordar a modelagem dos dados, os tipos de funções e as variáveis comumente empregadas para modelar a morte de árvores; e as formas como os diferentes modelos e sistemas empregam essas funções para simulação do crescimento e produção florestal.

Palavras-chave: Modelos; florestas nativas; simulação; predição.
\end{abstract}

\begin{abstract}
Natural forests mortality modelling. Natural forests are very complex ecosystems, for which frequently there are no data and adequate methods to describe growth and yield behaviour. Mortality, growth and recruitment are the basic components for forest modelling. The main objective of this paper is to provide a general overview of natural forest mortality modelling. It presents some aspects of the general occurrence, the mortality causes and types present in forests, the data acquisition and estimates based on forest inventories, the mortality rate values for different natural forests, the modelling approaches of mortality data, the functions and variables most commonly used, as well as how different models and systems use these functions for modelling growth and forest production. Keywords: Models; native forests; simulation; prediction.
\end{abstract}

\section{INTRODUÇÃO}

A floresta é um ecossistema complexo cuja estrutura e desenvolvimento envolvem muitos fatores. No dossel, a árvore compete com as plantas adjacentes por espaço, nutrientes e água, cercada da influência de insetos, doenças e intempéries. As árvores que sobrevivem durante um ano repetem esse processo até que cada uma sucumba à competição, à senilidade ou a fatores externos. Conhecer a probabilidade de sobrevivência de cada árvore é essencial para representar a progressão da floresta (BUCHMAN et al., 1983).

$\mathrm{O}$ entendimento das taxas e processos de mortalidade de árvores em todas as escalas contribui para o conhecimento dos sistemas naturais. Dados de mortalidade são necessários para avançar no entendimento da demografia das árvores. As taxas e distribuição da mortalidade são indicadoras da função do ecossistema. Um melhor entendimento das taxas de mortalidade em ecossistemas ajuda na detecção das pressões nos ecossistemas, causadas por poluição e outros distúrbios antropogênicos (CAREY et al., 1994). A taxa de eliminação natural em povoamentos florestais oferece um dos mais complexos problemas na mensuração florestal, qual seja, o conhecimento de quantas árvores em uma dada classe de DAP, em um determinado tempo, podem ser esperadas sobreviver durante um período de duração definida (DEEN, 1933). 
A mortalidade permanece como um dos menos compreendidos componentes das estimativas de crescimento de produção, principalmente por causa da complexidade do processo de mortalidade e da incerteza do tempo de ocorrência da morte das árvores (HAMILTON JR., 1986; YANG et al., 2003). Os processos fisiológicos envolvidos no crescimento e mortalidade de uma árvore individual e no desenvolvimento do povoamento são complexos. Apesar das pesquisas ecológicas e biológicas estarem continuamente avançando no entendimento qualitativo desses processos, a compreensão quantitativa é bem menos desenvolvida (YANG et al., 2003).

Um dos maiores interesses em pesquisa em biometria florestal é desenvolver modelos de crescimento e produção para a predição do crescimento de uma floresta ou de uma árvore individual. Um modelo típico compreende um componente de regeneração ou ingresso, um de crescimento ou incremento e um de mortalidade. Embora grandes avanços tenham sido feitos no desenvolvimento de equações de crescimento para árvores individuais ou povoamentos, para os componentes mortalidade e regeneração houve menos progressos (GUAN; GERTNER, 1991).

Infelizmente, poucos estudos foram desenvolvidos especificamente para aumentar o entendimento do processo de mortalidade. Poucos dos dados coletados para outros propósitos podem ser facilmente usados para o desenvolvimento de modelos de mortalidade ou sobrevivência. Na ausência de dados adequados, a mortalidade geralmente é tratada como uma suposição ou por julgamento subjetivo (HAMILTON JR., 1986).

Os modelos de crescimento de florestas são de grande valor quando usados na predição do desenvolvimento futuro do povoamento e, quando usados em conjunto com a análise econômica, ajudam a fornecer ao especialista florestal a base para a escolha de uma política ótima de manejo (RENNOLS; PEACE, 1986). De acordo com Vanclay (1983), muitos modelos de crescimento evitam o problema de predição da mortalidade por considerar que nenhuma mortalidade ocorre em uma plantação bem manejada. Essa suposição é razoável para plantios intensivamente manejados, mas é inapropriada para a modelagem de florestas naturais, onde a mortalidade é significativa e deve ser levada em consideração. Apesar da necessidade de predição da mortalidade, a maioria das publicações relativas a esse tema envolve povoamentos monoespecíficos e eqüiâneos, não sendo, portanto, adequados para a modelagem de florestas naturais (VANCLAY, 1991).

Existem poucos dados relativos à mortalidade em florestas tropicais não-manejadas. A ausência de anéis de crescimento torna difícil determinar as características básicas do povoamento, tal como idade e crescimento. A única maneira de estudar o crescimento e mortalidade é por meio de observações repetidas em parcelas permanentes de pesquisas em longo prazo, as quais são ainda raras nos trópicos (CAREY et al., 1994).

\section{Definição e ocorrência de mortalidade}

Com o desenvolvimento da ciência da ecologia no final do século XIX, pesquisas sobre padrões de mortalidade focaram primariamente as populações animais, enquanto que no início do século XX os ecologistas vegetais enfatizaram padrões da comunidade - o padrão de desenvolvimento de uma floresta no tempo, ou os padrões de vegetação ao longo de uma paisagem. Existia relativamente pouca ênfase nos processos demográficos que poderiam conter informações daqueles padrões. Análises iniciais das taxas de mortalidade de árvores partiram da suposição mais simples: a de que a chance de morte de uma árvore individual era independente de sua idade e dependente somente das características intrínsecas (genéticas) de sua espécie. Essa suposição resultava em uma curva de mortalidade que tinha a forma de uma curva exponencial negativa (BOTKIN, 1993).

Segundo Moser (1972), a mortalidade em povoamentos ineqüiâneos é largamente dependente da estrutura do povoamento. Povoamentos com grande número de árvores pequenas e com proporcionalmente menor número de árvores de grandes dimensões, terão taxas de mortalidade mais altas do que povoamentos com poucas árvores e com uma pequena razão numérica entre número de árvores nas sucessivas classes de diâmetro. Desconsiderando a contribuição do ingresso, um povoamento florestal irá gradualmente ser reduzido, em número, a uma taxa decrescente com o tempo, até que todas as árvores eventualmente morram. Como o povoamento inequiâneo é caracterizado por um decréscimo na freqüência de árvores à medida que os diâmetros das árvores aumentam, é esperado que as freqüências de mortalidade sejam maiores nas classes de diâmetro inferiores. Biologicamente, tal relacionamento é esperado, pois a mortalidade relacionada com a supressão aumenta com o aumento da freqüência de árvores. 
De acordo com Botkin et al. (1972a), o fundamento básico sobre a mortalidade é que a maioria das árvores das espécies dos estratos médios e superiores que não possam manter uma taxa mínima de crescimento provavelmente não sobrevivem por longo tempo na floresta. Além disso, existem outros eventos, tais como ventos, secas, raios, parasitismo e ataque de pragas, que podem resultar na morte de qualquer árvore em qualquer momento. Embora a floresta esteja em dinâmica contínua, há um equilíbrio em florestas naturais, onde as árvores mortas são continuamente substituídas por novos indivíduos. Em geral, as áreas de clareiras apresentam recrutamento superior à mortalidade; a fase de construção tende para o equilíbrio por um curto período, em seguida a mortalidade ultrapassa o ingresso; e na fase madura ocorre o equilíbrio dinâmico (CARVALHO, 1997). A morte de uma única árvore, ou um grupo de árvores do dossel, libera o espaço de crescimento disponível e cria uma clareira. Com o tempo, essa clareira será ocupada novamente pelo crescimento lateral das árvores circundantes, pelo aumento da regeneração ou por novo recrutamento (OLIVER; LARSON, 1996).

É difícil determinar exatamente quando a mortalidade ocorre. Em geral, o número máximo de árvores vivas em um povoamento é inversamente proporcional ao tamanho das árvores, conforme Reineke (1933), e o número máximo de árvores que um povoamento pode suportar diminui com a idade, já que as árvores constantemente ficam maiores. A morte pode ocorrer em períodos de déficit hídrico, quando as árvores suprimidas não estocaram suficientes nutrientes para sustentar o crescimento da parte aérea e das raízes. Árvores intolerantes ou aquelas de crescimento rápido em altura, com copas grandes ou que vivem em espaços restritos, sucumbem à mortalidade mais rapidamente, já que todos os fatores rapidamente reduzem a proporção de fotossíntese em relação à respiração (OLIVER; LARSON, 1996).

\section{Tipos de mortalidade}

Lee (1971) distinguiu dois tipos de mortalidade: a regular e a irregular. A mortalidade regular ocorre quando as árvores crescem tão próximas umas das outras que elas competem pela sobrevivência. Essa competição por limitado fornecimento de luz, água e nutrientes é mais intensiva em povoamentos densos ou superestocados e não ocorre com árvores que crescem em ambientes totalmente abertos. As árvores cobertas e suprimidas eventualmente morrem; já as árvores vigorosas e aquelas mais adaptadas ao ambiente tendem a ter maior probabilidade de sobrevivência. Essa probabilidade aumenta com o tamanho e idade até que a árvore esteja madura. Por outro lado, a mortalidade causada por danos de insetos, doenças, vento e fogo é chamada de mortalidade irregular. De acordo com Hann (1980), a mortalidade pode ser classificada em catastrófica (irregular) e não-catastrófica (regular). A morte catastrófica é o resultado de distúrbios em massa, normalmente imprevisíveis, como incêndios, tempestades, ventos, epidemias de doenças e ataques de insetos; já a mortalidade não-catastrófica acompanha o desenvolvimento normal do povoamento.

Lee (1971) usou a expressão "mortalidade regular" para mortes relacionadas à competição por recursos somente, enquanto Vanclay (1983) usa-a em um contexto mais amplo. O autor emprega a expressão "mortalidade regular" para referir a mortalidade que pode ser esperada em relativamente pequeno número de árvores (tanto indivíduos como pequenos grupos) em intervalos regulares. Esse tipo de mortalidade refere-se principalmente ao envelhecimento, supressão e competição, e também à mortalidade oriunda do acaso, ocorrência normal de pragas e doenças, e de condições climatológicas (secas, raios e tempestades), as quais comumente ocorrem (eventos com uma probabilidade de ocorrência de não menos que uma vez em dez anos). Já a mortalidade catastrófica inclui incêndios, grandes epidemias, ataques de pragas e perdas ocasionadas por condições climáticas severas (VANCLAY, 1994a). Vanclay (1983) também considera a mortalidade induzida pelo manejo, a qual inclui as mortes que ocorrem como resultado da interferência humana. Nesse caso, considera-se a mortalidade oriunda da extração madeireira, de tratamentos silviculturais aplicados na floresta e também dos danos diretos e indiretos da exploração florestal.

Robert (2003) considera em floresta tropical dois tipos de mortalidades naturais: a mortalidade primária e a secundária. A mortalidade primária é composta pela morte dependente da densidade do povoamento (morte da árvore em pé) e independente da densidade (queda de árvores). Essa mortalidade depende do estado de senilidade da árvore (representada pelo diâmetro), da competição a que a árvore tem sido submetida (representada pelo valor do crescimento prévio em diâmetro) e do estado de desequilíbrio causado pela assimetria da copa. A mortalidade secundária é relacionada àquelas árvores cujas quedas são induzidas pela queda de outras árvores. 


\section{Causas de mortalidade}

A análise e a comparação dos métodos para predição da mortalidade é facilitada com a adequada classificação da mortalidade de acordo com a causa e o padrão de ocorrência. Os padrões de mortalidade podem diferir em escala, freqüência e severidade, e isso deve ser considerado quando da escolha de uma estratégia de modelagem (VANCLAY, 1994a). Segundo Carvalho (1997), a mortalidade pode ser causada por diversos fatores, como: ataques de patógenos, parasitas e herbívoros; tempestades; danos causados por chuvas fortes, principalmente em árvores emergentes; danos durante a exploração e principalmente nos primeiros cinco anos seguintes; e morte por idade, considerando que todo ser vivo tem um período de vida finito. Em florestas tropicais, o padrão de mortalidade natural no tempo e no espaço está fortemente relacionado à máxima longevidade das árvores, distribuição em classes de tamanho, abundância relativa das espécies, e tamanho e número de aberturas no dossel da floresta.

A competição e a supressão são os agentes sobre os quais foi concentrada a maior atenção em modelagem florestal, especialmente em povoamentos puros. Reineke (1933) deu importantes contribuições ao entendimento do início da competição que induz a mortalidade em povoamentos eqüiâneos. Em florestas mistas, a situação é mais complexa e não existem teorias refinadas. Entretanto, a necessidade por luz, nutrientes e espaço físico é contínua e qualquer redução abaixo das necessidades mínimas irá eventualmente levar a árvore à morte (VANCLAY, 1983, 1994a).

Conforme Buchman (1979), a probabilidade da árvore morrer depende da sua habilidade para competir em seu ambiente. A árvore em si, as árvores vizinhas, e numerosas influências adicionais, incluindo luz, umidade, nutrientes, insetos e fungos, dentre outros, combinam para formar um nicho único para essa árvore individual. Já Lynch e Moser Jr. (1986) consideram que, como a área basal por hectare é algebricamente relacionada ao tamanho da árvore e ao número de árvores por hectare, a área basal associada a cada grupo de espécies tem um efeito sobre as taxas de mortalidade do grupo de espécies.

$\mathrm{O}$ efeito da produtividade do sítio sobre a sobrevivência não é claro. De acordo com Vanclay (1994a), existem evidências empíricas que em plantios a mortalidade dependente da densidade se expressa mais cedo nos melhores sítios, e se a mortalidade é expressa como uma função da idade, parece que a mortalidade aumenta com o aumento da produtividade do sítio. Entretanto, se a mortalidade é expressa em relação à altura do dossel ou densidade do povoamento, a situação torna-se diferente. Os melhores sítios deveriam ser hábeis para sustentar uma área basal mais alta, e mantendo-se constantes as demais variáveis, deveriam ter menor mortalidade.

Para Botkin et al. (1972a), os fatores bióticos e abióticos afetam cada espécie diferentemente e a possibilidade de sobrevivência em qualquer dado ano é determinada pela idade máxima conhecida de cada espécie. Vanclay (1994a) considera que a idade da árvore pode ser um fator contribuinte na mortalidade, mas pode não ser a causa da morte, e muitas mortes podem ser atribuídas à competição, pragas, doenças e eventos aleatórios. O tamanho da árvore não parece ser uma causa da morte, mas na prática é um bom preditor da probabilidade de mortalidade.

Vanclay (1994a) considera o clima como outro importante agente de mortalidade em povoamentos florestais. A ocorrência de secas ou inundações prolongadas pode acelerar a morte de árvores suprimidas, doentes ou velhas, e pode, ainda que raramente, causar a morte de árvores saudáveis sem a presença de tais agentes associados. Os raios e tempestades também podem matar ou danificar árvores. Os danos do vento podem ser catastróficos, e podem ou não ser dependentes das condições do povoamento. Os incêndios também são causas de perdas catastróficas. Entretanto, muitas outras causas de mortes de árvores podem ser atribuídas ao acaso, já que nenhuma explicação aparente pode ser encontrada.

A mortalidade causada diretamente por atividades humanas é também considerada por Vanclay (1983), que afirma que o mais importante aspecto da interferência humana é a exploração em que as árvores são removidas por causa da madeira. Outra importante atividade do manejo florestal é o tratamento silvicultural, em que os troncos indesejáveis são derrubados, anelados ou envenenados. Os danos originados pelas interferências humanas podem contribuir também com a mortalidade. A derrubada de árvores pode esmagar árvores menores, as máquinas podem danificar raízes e cascas e criar pontos de entrada para doenças e podridão, ou a exploração pode simplesmente alterar as condições do dossel e tornar o ambiente menos favorável a algumas espécies de árvores, ou mais favorável a doenças ou pragas. 


\section{Obtenção de dados de mortalidade}

A mortalidade natural é um evento raro, principalmente para árvores grandes não senis, que apresentam a taxa bem menor que $1 \%$ ao ano (WYCKOFF; CLARK, 2002). Por isso os dados são limitados, mesmo se houver disponível um grande conjunto de dados medidos. Além disso, geralmente, não há dados suficientes sobre as condições extremas da floresta por causa dos procedimentos típicos de seleção de parcelas (YANG; TITUS, 2002).

Carey et al. (1994) afirmam que o período de tempo é crítico em estudos de mortalidade de árvores, pois enquanto o crescimento é um processo constante, a morte é instantânea. Portanto, florestas estão sempre crescendo e acumulando biomassa em um dado intervalo de tempo, mas podem ou não perder biomassa por mortalidade no mesmo período de tempo, dependendo da área amostrada. Estudos de longo prazo são a única maneira de estimar com precisão a taxa de mortalidade em um determinado local.

De acordo com De Graaf et al. (1999), a mortalidade é mais complicada e tem custos mais altos para se estudar do que o incremento. As taxas de mortalidade anuais devem ser derivadas de populações de várias centenas de indivíduos observados em um longo período de tempo, de no mínimo dez anos, para compensar os eventos irregulares no clima e o ritmo de crescimento das árvores. Sheil (1995) afirma que existem diversos problemas em estimar a mortalidade das populações de árvores em inventários em parcelas permanentes. Um problema prático existente, conforme Kubo et al. (2000), que é comum em parcelas de grande tamanho, é a variável do intervalo de retorno entre os inventários das árvores. Normalmente leva um longo tempo para o estabelecimento de parcelas grandes, bem como para as remedições, tornando variável o intervalo de retorno para a remedição de cada árvore em cada espécie. Sheil (1995) afirma que nesses casos não se pode aplicar, por exemplo, uma estimativa de taxa de mortalidade instantânea (número de mortes por ano) entre dois inventários seqüenciais.

Sheil et al. (1995) afirmam que formulações mais complexas são necessárias para permitir a comparação de taxas de mortalidade obtidas em intervalos de tempo variados, porque essas medidas necessitam de conhecimento ou suposição de como as probabilidades de mortalidade variam com o tempo. Em muitas aplicações ecológicas essa probabilidade é considerada constante e é, portanto, usada para definir uma taxa de mortalidade.

Os métodos padrões de estimativas da taxa de mortalidade usados em florestas tropicais são baseados em suposições de que em uma população cada membro tem uma probabilidade igual e constante de morrer em uma unidade de tempo, gerando assim uma função exponencial negativa (SHEIL; MAY, 1996). Os problemas surgidos por tratar populações heterogêneas dessa maneira não têm sido largamente aceitos e tais erros têm sido chamados de tipo 3 por Sheil (1995). O autor afirma que esses erros não são causados pelo método de coleta de dados, nem pelo impacto sobre a área amostrada, mas pelas ocasiões em que os dados são inapropriadamente manipulados, analisados e/ou avaliados. O uso desses artifícios surge da limitada compreensão dos dados e dos sistemas sob estudo, e da necessidade de se fazer simplificações irreais e não comprovadas (SHEIL; MAY, 1996).

\section{Cálculo da taxa de mortalidade}

Os ecologistas freqüentemente medem e comparam as taxas de mortalidade e outras taxas dependentes de contagens. As medidas mais simples empregam contagens de mortalidade para populações pré-determinadas em um intervalo de tempo entre inventários definido (SHEIL et al., 1995).

A maioria dos estudos de mortalidade em florestas naturais emprega a função utilizada por Lieberman e Lieberman (1987), Manokaran e Kochummen (1987) e Swaine et al. (1987) para o cálculo da mortalidade anual. Essa função considera que a mortalidade decresce logaritmicamente com o tempo (LIEBERMAN; LIEBERMAN, 1987; SWAINE et al., 1987) e tem a forma:

$$
\lambda=\ln \left(N_{0} / N_{l}\right) / t
$$

Sendo: $N_{0}=$ número de árvores no primeiro inventário.

$N_{l}=$ número de árvores sobreviventes no segundo inventário $t$ anos depois.

Entre os estudos que utilizaram a função (1) citam-se Felfili (1993), Carey et al. (1994), Aiba e Kohyama (1996) e Köhler et al. (2001).

A aplicação do modelo CAFOGROM na Amazônia brasileira por Alder e Silva (2000) também empregou a função (1), porém utilizou períodos variados de intervalos de medição, usando para estimar a 
mortalidade uma aproximação linear com a forma $\Sigma t_{k}^{-1} / N$, em que $t_{k}$ é o intervalo de medição em anos para uma parcela e período durante o qual $k$ árvores morrem, e $N$ é o número total de observações (árvores x número de intervalos de medições), incluindo árvores vivas e mortas. A equação é derivada considerando-se que a mortalidade é uma função linear do tempo e é adequada à típica baixa taxa de mortalidade ( $1 \%-5 \%)$ aplicável a árvores em florestas naturais.

Tanto no estudo de Aiba e Kohyama (1996) em floresta pluvial temperada no sul do Japão como no de Köhler et al. (2001) empregando o modelo FORMIND1.1, em floresta pluvial tropical em Sabah, Malásia, os autores afirmaram que as árvores que recrutaram e morreram entre os inventários não foram consideradas no cálculo da taxa de mortalidade. Kubo et al. (2000) afirmaram que quando o intervalo de medição das árvores entre dois inventários é variável, não é possível aplicar estimativas da taxa de mortalidade instantânea (número de árvores que morreram no período de um ano) entre dois inventários seqüenciais, utilizando a função

$$
\left(\ln N_{0^{-}} \ln N_{1}\right) / t
$$

pelo fato do valor de $t$ não ser fixo. A taxa de mortalidade é uma estimativa da probabilidade no nível da população, e o método da máxima verossimilhança é então aplicável para sua estimativa, usando-se todos os registros dos intervalos dos inventários para cada árvore. Os autores compararam a taxa de mortalidade calculada pela função (2) e pelo método da máxima verossimilhança, concluindo que a diferença entre as duas estimativas foi menor que $3 \%$. Entretanto, essa diferença era maior com a redução do tamanho da população e com o aumento da mortalidade.

Pelo fato de que as taxas de mortalidade são baixas e de que seria necessária uma grande amostra para obter essa taxa de forma adequada usando técnicas padrão de regressão binomial, Pacala et al. (1993) desenvolveram uma alternativa para o submodelo de mortalidade no modelo SORTIE. Essa metodologia requer a taxa de crescimento de uma amostra de somente 35 a 50 indivíduos mortos em pé e 35 a 50 indivíduos vivos, e uma contagem de árvores vivas e mortas ao longo de transectos.

Sheil et al. (1995) examinaram algumas discrepâncias que ocorrem na literatura relacionada ao uso da função do cálculo da mortalidade em povoamentos florestais. Na forma mais simples, a mortalidade constante $(\lambda)$ é representada pelo declínio exponencial da população como:

$$
\begin{aligned}
& \mathrm{d} N / \mathrm{d} t=-\lambda N \\
& \lambda=\ln \left(N_{0} / N_{l}\right) t .
\end{aligned}
$$

Essa é a forma da função mais empregada para o cálculo da taxa de mortalidade. Outra formulação algébrica de mortalidade é o uso da mortalidade anual $(m)$ como uma taxa variável:

$$
N_{l}=N_{0}(1-m)^{t}
$$

podendo, então, a mortalidade ser calculada como

$$
m=1-\left(N_{1} / N_{0}\right)^{1 / t}
$$

ou ainda, se for usada a contagem do número de árvores perdidas $\left(N_{0}-N_{l}\right)$, assim:

$$
m=1-\left[1-\left(N_{0}-N_{1}\right) / N_{0}\right]^{1 / t}
$$

Segundo os autores, o problema surge quando se comparam as mortalidades de diferentes florestas calculadas $\operatorname{com} \lambda$ e $\operatorname{com} m$. As duas medidas são independentes do tempo e a equivalência das duas pode ser obtida como:

$$
\begin{aligned}
& \lambda=-\ln (1-m) \\
& \mathrm{e} \\
& m=1-e^{-\lambda}
\end{aligned}
$$

Como $\lambda$ é uma medida instantânea do declínio por unidade da população, não deveria ser considerada como mortalidade anual. A verdadeira taxa anual é definida pela variável $m$. $\mathrm{O}$ valor $\lambda$ deveria ser referido como um coeficiente de mortalidade exponencial. Na realidade, as discrepâncias são 
pequenas, exceto em altas taxas de mortalidade. Por exemplo, para mortalidade de $2 \%$, a discrepância é de $0,02 \%$, enquanto que para $50 \%$ a discrepância aumenta para $19 \%$. Quando é afirmado que a taxa de mortalidade anual é derivada de acordo com o modelo exponencial, essa informação não é suficiente para distinguir a variável $\lambda$ da variável $m$.

\section{Modelagem de mortalidade}

O entendimento dos processos biológicos associados com o crescimento e mortalidade das árvores pode ajudar a modelagem de duas maneiras. Primeiro, auxilia a formular hipóteses entre os problemas de mortalidade e as variáveis independentes disponíveis. Segundo, é útil para a própria avaliação e interpretação de formas de modelo alternativas. Qualquer relação modelada que viola os princípios biológicos deve ser rejeitada, mesmo se resulta em predições eficientes da mortalidade para as condições de um conjunto de dados (HAMILTON JR., 1990).

De acordo com Vanclay (1989), diversas abordagens para predizer a mortalidade têm sido descritas, mas a maioria foi desenvolvida para povoamentos monoespecíficos e não é adequada para modelagem de povoamentos de florestas naturais. Os procedimentos de densidade de povoamento, como o de Reineke (1933), indicam somente o estoque residual e não dão indicação de quais árvores morrem.

Os algoritmos de mortalidade variam em sua forma e parâmetros, mas todos eles são derivados de uma premissa básica que determina que, além de um especificado ponto, uma planta (ou uma porcentagem da comunidade) ou morre (estrutura determinística) ou tem um aumento na probabilidade de morrer (estrutura estocástica). Os algoritmos podem potencialmente incorporar tanto elementos estocásticos como determinísticos, e um modelo poderia incluir tanto um algoritmo de mortalidade determinístico quanto estocástico (HAWKES, 2000). Conforme Gertner e Dzialowy (1984), com um modelo estocástico pode-se estimar a variância, ao contrário do determinístico, e os coeficientes são determinados com os procedimentos habituais de regressão não-linear.

A implementação estocástica necessita de um número aleatório que é comparado com a probabilidade predita da mortalidade. Alternativamente, essas probabilidades podem ser interpretadas como proporções, e o estoque em cada grupo ou classe pode ser reduzido pela proporção predita. Esse procedimento alternativo deveria produzir predições compatíveis, porém existem vantagens computacionais em simular a mortalidade de uma forma determinística, a não ser que o usuário esteja especificamente interessado em estudos de variabilidade (VANCLAY, 1994a). As predições determinísticas da mortalidade são obtidas quando um valor específico de uma determinada variável é ultrapassado (quando um valor observado é maior do que o valor limite predeterminado). Diferentemente das predições de uma variável contínua como o crescimento, em que a biomassa pode aumentar $x$ vezes além de um limite, na mortalidade determinística existe somente um limite que define duas classes: vivas ou mortas (HAWKES, 2000).

Os algoritmos estocásticos incorporam aleatoriedade no processo de mortalidade, enfatizando a variabilidade natural e a incerteza na morte de árvores. Quando o algoritmo é estocástico, cada valor do preditor usado tem uma probabilidade associada de mortalidade que varia de 0 a 1 . A relação entre eles pode tomar diferentes formas funcionais. Em uma simulação, a cada iteração, a probabilidade associada com o valor do preditor é comparada com um número aleatório entre 0 e 1 . Para modelos baseados em indivíduos, se a probabilidade for maior do que o número aleatório, a árvore é considerada morta (HAWKES, 2000).

Hawkes (2000) analisou mais de 150 modelos de crescimento, mostrando que menos da metade deles (61 modelos) incluíam um algoritmo de mortalidade, e que a maioria desses algoritmos eram empíricos (ajustados estatisticamente), sendo que poucos eram baseados em processos. Segundo Weber et al. (1986), muitos modelos de crescimento de povoamentos florestais baseados em árvores individuais descrevem a mortalidade incluindo termos aleatórios para processos que são em parte estocásticos. Entretanto, poucos estudos têm comentado sobre o comportamento estocástico resultante dos modelos ou quando um procedimento estocástico deveria ser usado no lugar de um procedimento determinístico. Uma limitação dos processos estocásticos é que podem requerer repetidas vezes a execução para obter uma média de resultados para a análise ser significativa. Uma maneira de aumentar a eficiência de tais modelos de crescimento é a técnica da redução da variância ou método de Monte Carlo.

Para esclarecer essa escolha no procedimento de estimar a mortalidade, Weber et al. (1986) compararam a projeção em longo prazo usando processos de estimativas da mortalidade estocásticos e determinísticos. Os resultados confirmaram a eficiência das técnicas de estimar a mortalidade 
deterministicamente. Em relação à eficiência no emprego adicional do modelo, sugeriram o uso de algoritmos determinísticos para a mortalidade, a não ser que o interesse resida em estudo de variabilidades e implicações relacionadas no desenvolvimento de povoamentos florestais.

\section{Funções para modelagem de mortalidade}

De acordo com Yang et al. (2003), as funções lineares e polinomiais foram comumente usadas para modelar a mortalidade nas décadas de 60 e 70 do século XX, para modelos ajustados estatisticamente. Esses modelos de mortalidade podem funcionar bem dentro dos limites de dados para os quais foram desenvolvidos. Entretanto, as formas funcionais são freqüentemente não-compatíveis com o comportamento biológico e ecológico do crescimento de árvores e mortalidade. Nesse caso, a probabilidade predita da mortalidade não é garantida a permanecer nos limites $(0,1)$, o qual é um requisito crítico para modelagem da mortalidade. Existem muitas funções não-lineares que são implicitamente definidas entre zero e um. Entre elas, as distribuições de probabilidade cumulativa, incluindo a distribuição binomial negativa, a distribuição Weibull e a distribuição gama podem ser usadas para modelar a mortalidade.

A maior parte dos modelos de classe de tamanho usam um método empírico para predizer a mortalidade, freqüentemente como funções lineares simples da densidade do povoamento e tamanho relativo. Tais funções deveriam ser usadas com cautela, já que elas podem dar estimativas fora dos limites possíveis $(0,1)$, se usadas além dos limites de dados do qual foram desenvolvidas (VANCLAY, 1994b). Entretanto, muitas formas de modelos forçam para que as probabilidades fiquem no intervalo de 0 a 1 (BUCHMAN et al., 1983).

Entre os vários métodos estatísticos disponíveis que podem ser usados para desenvolver uma função empírica de mortalidade individual de árvores, a regressão logística é a mais largamente empregada e provavelmente o melhor método disponível (GUAN; GERTNER, 1991). Como sugerido por Hamilton e Edwards (1976), na regressão logística, os valores preditos ficam limitados entre 0 e 1 e a forma da função é uma escolha biologicamente preferível para modelagem da mortalidade de árvores individuais. A limitação dos valores entre 0 e 1 caracteriza a variável dependente como dicotômica e produz modelos com propriedades estatísticas melhoradas, quando comparada com os métodos lineares de mínimos quadrados (HANN, 1980).

Vanclay (1995) acredita que a função logística ajustada a dados individuais de árvores pode oferecer a melhor maneira de modelar a mortalidade em florestas tropicais, a qual cobre um espectro de mistura de diferentes espécies e idades, não exigindo o uso da idade do povoamento ou do índice de sítio como variáveis preditoras. Hamilton Jr. (1986) cita vantagens específicas da função logística: a) como a função é limitada entre 0 e 1 , ela é naturalmente limitada à amplitude do potencial da probabilidade de mortalidade ou sobrevivência; b) com uma seleção do conjunto próprio de variáveis e transformações, a função logística pode ser usada para descrever a maioria dos padrões de mortalidade que ocorrem naturalmente; e c) rotinas de estimativas não-lineares (tanto rotinas de mínimos quadrados como de máxima verossimilhança) podem estimar eficientemente os parâmetros da função logística, e esta eficiência não parece ser afetada pela seleção de valores iniciais.

Segundo Vanclay (1994a), a função logística pode ser expressa de várias maneiras, como:

$$
P=\left(1+e^{-f(x)}\right)^{-1} \quad(10) ; P=1-\left(1+e^{f(x)}\right)^{-1} \quad(11) ; \text { ou } P=\frac{e^{f(x)}}{1+e^{f(x)}}
$$

Sendo: $P=$ probabilidade de sobrevivência.

$f(x)=$ função (freqüentemente linear) de diversas variáveis explicativas.

A probabilidade de mortalidade pode, então, ser obtida de (1-P). A vantagem de predizer a sobrevivência é que ela, diferente da mortalidade, é um processo de Markov (isto é, é um estado transitório em vez de um estado absorvente), tal que a sobrevivência em um período de $n$ anos é dada pela $n$-ésima potência da probabilidade anual de sobrevivência. Diferentes intervalos de medida podem ser acomodados pelo uso do intervalo como um expoente (isto é, -t pode substituir -1 na equação acima). Isso pode algumas vezes causar dificuldades, e uma eficiente alternativa é ponderar a equação acima pelo intervalo de remedição (HAMILTON JR.; EDWARDS, 1976). Ainda que não tecnicamente correto, essa 
alternativa parece aceitável, uma vez que remedições são regularmente freqüentes e a mortalidade é relativamente baixa. Se os dados estão agrupados em categorias com, no mínimo, uma árvore morta e uma viva em cada categoria, a equação (12) pode ser convertida em uma equação linear da forma:

$$
y=\ln \left(\frac{P}{1-P}\right)=\ln \left(\frac{N_{\text {sobrev }}}{N_{\text {mortas }}}\right)=f(X)
$$

Sendo: $\quad N_{\text {sobrev }}=$ número de árvores sobreviventes.

$N_{\text {mortas }}=$ número de árvores mortas.

A vantagem de usar a equação (11) sem transformação é que permite o uso de dados individuais, possibilitando extrair máxima informação, não havendo necessidade de usar o ponto médio da classe para o tamanho das árvores e para outras variáveis explicativas (VANCLAY, 1983, 1994a).

\section{Variáveis nos modelos de mortalidade}

Monserud (1976) usou como variáveis independentes no modelo de mortalidade o diâmetro predito, o crescimento predito do diâmetro e um índice de competição. Os modelos de mortalidade no sistema PROGNOSIS 4.0 foram baseados primariamente no diâmetro (DAP e DAP ${ }^{2}$ ), sendo que a influência da densidade do povoamento sobre a probabilidade de mortalidade foi restrita aos povoamentos jovens e aos povoamentos próximos de atingir a máxima área basal (WYKOFF et al., 1982). Já na versão 5.0 (WYKOFF, 1986), o modelo de mortalidade incorporou estimativas preditas de duas equações independentes. Essas estimativas eram baseadas em tipo de hábitat, espécies, DAP, incremento do DAP, estimativa potencial de incremento do DAP, área basal do povoamento e DAP relativo (DAP/DAP médio do povoamento).

Botkin et al. (1972b) fizeram a predição de mortalidade para qualquer árvore como uma função da idade máxima potencial da espécie. Ek e Monserud (1974) usaram uma função exponencial da razão da copa, comprimento do período de crescimento e um índice de competição para especificar uma função da mortalidade. Hamilton Jr. e Edwards (1976) desenvolveram um método para predição individual de mortalidade de árvore como uma função somente do DAP da árvore. Hamilton Jr. (1986) afirmou que a variação na mortalidade de árvores pode freqüentemente ser explicada por uma medida do tamanho da árvore, densidade do povoamento, competição individual e taxa de crescimento da árvore.

De acordo com Buchman (1979), um dos mais simples indicadores das condições das árvores é o vigor, refletido no crescimento do diâmetro, sendo uma característica chave nas funções de mortalidade. $\mathrm{O}$ autor estimou a taxa de sobrevivência anual a partir do tamanho e vigor da árvore no ano anterior, sendo o DAP e a taxa de crescimento do diâmetro representantes dessas duas características.

As variáveis independentes usadas por Hann (1980) foram transformações a partir da classe de diâmetro ou a partir de atributos do povoamento e incorporadas não somente aos fatores correlacionados à mortalidade, mas também a fatores que hipoteticamente poderiam estar correlacionados à morte de árvores. Inclui-se nesse grupo o tempo desde o último corte e o crescimento da área basal predito a partir dos três modelos. A afirmação de Kohyama (1993) de que a probabilidade de morte instantânea de árvores seria provavelmente uma função do aumento de densidade $B(x)$ do local, não pode ser comprovada com os dados obtidos de uma floresta tropical de dipterocarpáceas da Indonésia. A mortalidade foi, então, descrita como simplesmente dependente do tamanho das árvores (KOHYAMA et al., 2001).

\section{Dificuldades na modelagem da mortalidade}

$\mathrm{Na}$ maioria das florestas naturais, principalmente tropicais, é impraticável obter funções de mortalidade individuais para cada espécie, por causa do grande número de espécies e do reduzido número de dados (baixa abundância) para muitas delas, o que impediria o desenvolvimento de relações confiáveis. Assim, para uma estimativa eficiente de funções de mortalidade, é desejável agregar espécies em vários grupos. Idealmente, as espécies deveriam ser agrupadas e testadas para justificar a validade de tais agrupamentos. Isso pode ser possível em florestas temperadas, onde existem poucas espécies e sua ecologia é bem documentada, porém é impossível em floresta tropical úmida, onde há centenas de espécies, muitas das quais não bem conhecidas. A mortalidade pode ser dependente do tamanho da árvore e densidade do povoamento, tal que o agrupamento baseado na mortalidade média poderia ser específico para o conjunto de dados utilizado (VANCLAY, 1991). 
Stage e Renner (1988) indicaram que a maioria (80 \%) da variabilidade na predição de volume em florestas mistas de coníferas nas Montanhas Rochosas, Estados Unidos, era causada pelas incertezas nas estimativas da mortalidade. Claramente as estimativas de mortalidade podem ter uma grande influência na acuracidade das previsões de produção e crescimento. Hawkes (2000) cita dificuldades e problemas encontrados para a modelagem da mortalidade. Entre eles, podem ser citados: a) existem vários fatores abióticos e bióticos que afetam a mortalidade; b) há uma limitada compreensão do processo de mortalidade nas plantas; c) praticamente todos os algoritmos de mortalidade necessitam de relativamente grande número de dados; d) muitos algoritmos não levam em conta as diferenças de espécies; e) usualmente considera-se que a mortalidade é uma resposta instantânea ao estresse, ao contrário de ser um resultado de formação cumulativa de estresse; f) quase sempre é difícil identificar fontes e quantidades de erros; e g) os erros são abundantes na simulação da mortalidade, na construção e na estrutura do modelo, por causa da qualidade e quantidade disponível de dados.

\section{Mortalidade em modelos e sistemas}

De acordo com Bugmann (1996), em FORCLIM-P (Plantas), um dos três submodelos do modelo FORCLIM (modelo sucessor do FORECE), o estabelecimento e crescimento das árvores são modelados em grupos, enquanto que as funções de mortalidade são avaliadas para cada membro de cada grupo de árvores individualmente. $O$ submodelo considera que existem duas fontes de mortalidade: 1) a mortalidade é calculada como um processo de Poisson, supondo que a taxa anual é constante ao longo da vida da árvore, correspondendo a uma curva exponencial negativa para a sobrevivência. A função é escalar, de tal forma que $1 \%$ das árvores alcança a idade específica da espécie; 2) a taxa de mortalidade induzida por estresse é aplicada quando o DAP de uma árvore aumenta menos do que $10 \%$ do incremento máximo em DAP ou quando o incremento for menor que $0,3 \mathrm{~mm}$, por dois anos ou mais. A função também é escalar, tal que somente $1 \%$ das árvores deverão sobreviver por dez anos se permanecerem sujeitas a essa taxa de crescimento.

A probabilidade de mortalidade global é então obtida para cada membro dos grupos de árvores pelos dois componentes acima citados. Dessa forma, além do valor do DAP, é introduzida uma segunda variável de estado por grupo de árvores no modelo que é obtido a partir do número de anos em que uma árvore cresce lentamente (BUGMANN, 1996). Kohyama (1992), usando dados de parcelas permanentes em floresta temperada no sul do Japão, modelou a mortalidade de árvores por espécies em função do tamanho e da intensidade de "sombreamento", que é a área basal cumulativa das árvores maiores que a árvore alvo. Entretanto, não houve dados suficientes para se obter uma função empírica. Assim, foi considerada a mortalidade simplesmente como uma constante específica da espécie independente do tempo e do tamanho da árvore. Em outro estudo em floresta tropical em Kalimantan (Indonésia), Kohyama et al. (2001) também não detectaram a dependência da mortalidade em relação à superpopulação representada pela variável $B(x)$, o índice de sombreamento. Assim, foi empregada a função:

$$
D=c+c_{1} / x
$$

dependente apenas do tamanho da árvore, sendo que o parâmetro $c$ (ano ${ }^{-1}$ ) é a mortalidade assintótica para árvores grandes, e é usado para fornecer a taxa de formação de clareiras. O outro parâmetro, $\mathrm{c}_{1}\left(\mathrm{~cm} \mathrm{ano}^{-1}\right)$, descreve o grau de mortalidade adicional para as árvores pequenas.

Kohyama (1991), com dados de floresta tropical da Sumatra (Indonésia) e de floresta pluvial temperada do sul do Japão, empregou a função:

$$
M(t, x)=c+c_{1} / x
$$

para simulação da mortalidade $M(t, x)$ em árvores de tamanho $x$ no tempo $t$, que define a mortalidade como independente de $B$ (área basal cumulativa das árvores maiores que $x$ ). Em floresta tropical, a mortalidade foi então tratada como independente do DAP, apesar de que a variável $M(t, x)$ implicitamente incorpora a mortalidade por supressão. Quando Kohyama (1989) usou o modelo linear:

$$
M=a+b * B
$$

o qual relaciona $B$ e $M(t, x)$ para incorporar o efeito da supressão na mortalidade, os resultados da simulação para floresta pluvial temperada mostraram pouca diferença do modelo básico. 
No sistema JABOWA, um simulador estocástico que tenta reproduzir as características aleatórias e probabilísticas de uma floresta, desenvolvido por Botkin et al. (1970), a mortalidade é considerada na sub-rotina KILL, na qual é decidido estocasticamente quais árvores morrerão na simulação. A morte da árvore pode ocorrer por dois mecanismos separados. O primeiro é uma função da idade máxima conhecida da espécie $(A G E M X)$. A suposição é que não mais de $2 \%$ das árvores de uma espécie alcançaria a idade máxima. Se a probabilidade de morte é independente da idade de uma árvore, a probabilidade de que a árvore irá morrer em algum ano é:

$$
p=1-(1-\varepsilon)^{n}
$$

Se $p=0,98$ quando $n=A G E M X$, então $(1-\varepsilon)^{A G E M X}=2 \times 10^{-2}$, ou aproximadamente $\varepsilon=4,0 / A G E M X$. O programa gera um número aleatório entre 0 e 1 , e se esse número for menor ou igual ao valor de $\varepsilon$, a árvore está morta. Se a árvore não for removida por esse método, a sub-rotina determina se o último incremento em diâmetro foi maior que um valor mínimo, por exemplo 0,01 cm (BOTKIN et al., 1972a). $\mathrm{O}$ sistema considera que uma árvore cujo incremento de diâmetro fique abaixo desse valor mínimo por dez anos sucessivos não teria chance maior de $1 \%$ de sobreviver nesse período (BOTKIN et al., 1972b).

O modelo de sucessão OUTENIQUA foi desenvolvido por van Daalen e Shugart (1989) para as florestas mistas da África do Sul. Da mesma forma que o modelo JABOWA, os autores usaram a proporção de árvores do grupo que alcançam a idade máxima, porém definiram a taxa em somente $1 \%$. E o incremento em diâmetro mínimo necessário para garantir a sobrevivência da árvore foi de $0,25 \mathrm{~mm}$ ano${ }^{1}$ (0,01 cm no JABOWA). A probabilidade também foi obtida em função da idade máxima.

Belcher et al. (1982) desenvolveram o modelo STEMS para projeção de crescimento de árvores em povoamentos baseado em princípios biológicos. O processo da mortalidade natural é simulado pela aplicação da função desenvolvida por Buchman (1979), que prediz a probabilidade de morte de um indivíduo em um ano como uma função do DAP atual da árvore e da taxa de crescimento anual, na forma de um modelo logístico. Em STEMS, a mortalidade individual pode ser probabilística ou determinística. $\mathrm{Na}$ primeira, a definição das árvores consideradas mortas é feita com base na comparação com um número aleatório, e na segunda alternativa o número de árvores por hectare que essa árvore representa é reduzido pela proporção predita pela função de mortalidade.

Em estudo da mortalidade em florestas de folhosas no baixo Mississipi, Zhao et al. (2004) usaram uma função logística para cada grupo de espécies, selecionando os preditores entre oito variáveis independentes: DAP, 1/DAP, DAP ${ }^{2}$, DAP relativo, DAP quadrático relativo, área basal, área basal relativa da espécie e classe de copa. Os dados foram tomados com base em períodos de observação de cinco anos. O método da máxima verossimilhança foi usado para estimar os parâmetros desconhecidos, e o melhor modelo para cada grupo de espécie foi selecionado baseado no teste estatístico de aderência do ajuste de Hosmer-Lemeshow e no comportamento ecológico do modelo ajustado.

Köhler et al. (2001) estudaram a mortalidade em parcelas permanentes em floresta pluvial de dipterocarpáceas na Malásia, utilizando o modelo FORMIND1.1. A mortalidade foi modelada com base anual e não depende de outros processos, como o incremento em diâmetro. O modelo inclui uma mortalidade adicional de superpopulação para árvores em áreas densas (onde as copas não têm espaço suficiente). Nesse caso, as árvores morrem em grande número e a superpopulação não ocorre mais. Por causa do curto tempo das simulações (menor ou igual a 20 anos) não foram incluídos processos de queda de árvores e a criação de clareiras por essas árvores.

O modelo FORMIND2.0 foi empregado para modelar o crescimento e a produção de floresta explorada na Venezuela. A mortalidade foi modelada estocasticamente com base na taxa básica anual e correlacionada à taxa de crescimento do diâmetro e ao tamanho máximo do DAP. A isso foi adicionada uma mortalidade dependente do diâmetro, a qual é zero acima do diâmetro limite considerado (KAMMESHEIDT et al., 2001).

Namaalwa et al. (2005) construíram um modelo de crescimento dependente da densidade para as florestas áridas de Uganda. Na função de mortalidade, a probabilidade de que uma árvore de uma determinada espécie e classe diamétrica morra entre dois inventários foi modelada por uma função logística, na qual são incluídas três variáveis, representando o tamanho das árvores, a densidade e a estrutura do povoamento. A mortalidade diminuiu com o aumento do DAP e aumentou com o aumento da área basal, estando esses efeitos de acordo com as expectativas biológicas. 
Khatouri e Dennis (1990) desenvolveram o modelo CEDGY para povoamentos dominados por Cedrus atlantica no Marrocos. Para a modelagem da mortalidade, os autores combinaram o produto de duas funções não-lineares. A primeira é uma função logística para estimar a ocorrência de morte como uma função da densidade de árvores (número por hectare). A segunda é uma função não linear ajustada ao conjunto de dados contendo somente as parcelas que tiveram mortalidade durante o último período de dez anos, para predizer a mudança no número de árvores mortas. Nessa última função, foi incluída como variável independente, além do número de árvores, a área basal total por hectare no início do período de medição. $\mathrm{O}$ modelo de mortalidade subestimou a taxa de mortalidade observada, e isso provavelmente ocorreu pela dificuldade de estimativas da mortalidade em parcelas temporárias.

Phillips et al. (2003) aplicaram o sistema SYMFOR em florestas de dipterocarpáceas em Kalimantan, Indonésia, no qual a probabilidade de mortalidade de árvores individuais foi modelada com base anual como uma função do diâmetro (DAP). Isso representa um aumento linear na probabilidade de mortalidade com o incremento do diâmetro até um diâmetro limite determinado para cada grupo de espécie, acima do qual a mortalidade é aumentada por um fator de 10. A probabilidade então é comparada com um número aleatório, e se for maior que esse número, a árvore é considerada morta. O modelo de mortalidade interage com o termo de correlação das taxas de crescimento modeladas de árvores grandes. Assim, árvores que crescem mais lentamente tendem a morrer antes de se tornarem grandes. Os danos resultantes da queda são calculados considerando a área afetada pela queda, como um trapézio dependente do tamanho da árvore.

\section{Taxas de mortalidade em florestas naturais}

Os valores típicos das taxas médias de mortalidade de árvores em florestas tropicais primárias são de $1 \%$ a $2 \%$ ao ano, com uma significante taxa mais alta para espécies pioneiras (KÖHLER et al., 2001). Phillips et al. (1994) compilaram dados sobre taxas anuais de mortalidade de florestas tropicais em 25 sítios localizados em quatro continentes. As taxas variaram de $0,67 \%$ a $2,85 \%$, sendo as mais altas encontradas na Amazônia peruana e as mais baixas em Queensland (Austrália) e em Uganda.

Em florestas de dipterocarpáceas na Malásia, Manokaran e Kochummen (1987) encontraram taxa de mortalidade média anual de $2,02 \%$ em um período de observação de 34 anos, sendo que as árvores emergentes apresentaram taxa mais baixa $(1,4 \%)$, enquanto que as árvores do sub-bosque atingiram mortalidade de 2,6 \%. Köhler et al. (2001) estudando florestas pluviais em Sabah, Malásia, em períodos de 9 a 20 anos, obtiveram taxas de mortalidade anual variando de $0,24 \%$ a $6,34 \%$, em distintos locais, sendo as taxas de mortalidade de espécies pioneiras geralmente maior do que as de espécies não pioneiras, alcançando $12,03 \%$. Em relação ao tempo, as taxas médias mostraram alta flutuação, podendo em alguns casos mostrar $0 \%$ nos oito primeiros anos e $36 \%$ no último período analisado.

Em floresta primária tropical úmida em La Selva, Costa Rica, Lieberman e Lieberman (1987) encontraram, em 14 anos de observações, uma taxa média de mortalidade anual de 2,03\%. Também na Costa Rica, em floresta explorada, Finegan e Camacho (1999) observaram taxas entre 1,6 \% e 2,3 \% ao ano, sendo que as árvores com incremento em diâmetro zero ou negativo tiveram marcadamente maiores taxas de mortalidade do que aquelas árvores com incremento anual médio maior ou igual a 1,0 $\mathrm{mm}$. Notadamente, a mortalidade aumentou com a redução da iluminação da copa. Em estudo em uma floresta na Amazônia 13 anos após a exploração, Silva et al. (1995) obtiveram taxa anual de mortalidade de 2,2 \% para todas as espécies e de 1,3\% para as espécies comerciais. Castro Marín et al. (2005), em floresta decídua seca e em floresta de galeria na Nicarágua, encontraram taxas de mortalidade de $4,5 \%$ e 4,2 \%, respectivamente, durante sete anos de estudos. Felfili (1993) estudou a dinâmica de uma floresta de galeria em área de cerrado próximo a Brasília, obtendo taxa de mortalidade de 3,5\% em seis anos de observações. A competição entre as pequenas árvores e a senilidade entre as árvores maiores foram provavelmente as principais causas de morte.

Em estudos na floresta amazônica brasileira, Alder e Silva (2000) encontraram que, em média, as árvores dominadas têm mortalidade $1,7 \mathrm{vez}$ maior do que as dominantes, e que árvores com defeitos tem mortalidade 2,6 vezes maior. Para nenhum grupo de espécies a mortalidade de árvores dominantes foi maior do que as dominadas, e para as dominantes a taxa variou de $0,5 \%$ a $4,1 \%$ ao ano. Os autores citam que as taxas mais altas de mortalidade estão associadas com as espécies que tendem a ter maior necessidade de luz (pioneiras). 
Pulz (1998) estudou a dinâmica de uma floresta semidecídua montana no Estado de Minas Gerais em períodos de quatro, cinco e nove anos, obtendo taxas anuais de mortalidade de 3,6, 2,0 e 2,3 \%, respectivamente. A mortalidade concentrou-se nas classes diamétricas menores e nas espécies exigentes de luz, chegando nestas últimas a uma taxa de 10,6\%. A mortalidade encontrada em uma floresta decidual estacional localizada na região central do Estado do Rio Grande do Sul foi de 4,8 \% em três anos de observações. As árvores pequenas do estrato inferior mostraram uma taxa de mortalidade maior do que as emergentes, causada provavelmente pela competição, não sendo encontrada nenhuma mortalidade de árvores acima de $52,5 \mathrm{~cm}$ de DAP. Os autores também obtiveram grande diferença de mortalidade entre as espécies (SPATHELF; DURLO, 2001).

Sanquetta et al. (2003) compararam a dinâmica em duas florestas com araucária (Ombrófila Mista) localizadas no sul e sudeste do estado do Paraná (municípios de General Carneiro e São João do Triunfo). As observações realizadas durante um período de quatro e seis anos mostraram, respectivamente, taxas de mortalidade $1,01 \%$ e de $1,9 \%$, sendo que a maior taxa foi atribuída à competição das árvores pela maior densidade da floresta. Considerando somente a espécie Araucaria angustifolia, a mortalidade foi de $0,19 \%$ e $0,33 \%$, respectivamente.

\section{CONSIDERAÇÕES FINAIS}

O fato de a ocorrência da mortalidade de árvores em florestas naturais ser geralmente um evento raro dificulta a obtenção de dados adequados e suficientes para a modelagem, mesmo com o uso de observações em parcelas permanentes. A irregularidade da mortalidade tanto temporal quanto espacialmente exige observações em parcelas de tamanho maior e por períodos de tempo mais longos, o que é normalmente possível somente em estudos científicos.

$\mathrm{Na}$ modelagem da mortalidade, a função logística parece ser a função mais adequada e flexível para predição do número de árvores mortas em povoamentos ineqüiâneos. As variáveis independentes que possuem maior influência na mortalidade são a taxa de incremento e a densidade do povoamento, sendo também fatores importantes a ocorrência de pragas, doenças, incêndios, ventos e os fatores antropogênicos, principalmente extração madeireira e tratamentos silviculturais nas florestas manejadas.

\section{REFERÊNCIAS}

AIBA, S.; KOHYAMA, T. Tree species stratification in relation to allometry and demography in a warmtemperate rain forest. Journal of Ecology, Oxford, v. 84, n. 2, p. 207-218, 1996.

ALDER, D.; SILVA, J. N. M. An empirical cohort model for management of Terra Firme forests in the Brazilian Amazon. Forest Ecology and Management, Amsterdam, v. 130, n. 1-3, p. 141-157, 2000.

BELCHER, D. W.; HOLDAWAY, M. R.; BRAND, G. J. A description of STEMS: the stand and tree evaluation and modeling system. Saint Paul: Forest Service/North Central Forest Experiment Station, 1982. 18 p. (General Technical Report, n. 79)

BOTKIN, D. B. Forest Dynamics: an ecological model. Oxford: Oxford University Press, 1993. 309 p.

BOTKIN, D. B.; JANAK, J. F.; WALLIS, J. R. A simulator for northeastern forest growth: a contribution of the Hubard Brook Ecosystem study and IBM research. IBM Research Report, Armonk, n. 3140, p. 1$21,1970$.

BOTKIN, D. B.; JANAK, J. F.; WALLIS, J. R. Rationale, limitations, and assumptions of a northeastern forest growth simulator. IBM Journal of Research and Development, Armonk, v. 16, n. 2, p. 101-116, 1972a.

BOTKIN, D. B.; JANAK, J. F.; WALLIS, J. R. Some ecological consequences of a computer model of forest growth. Journal of Ecology, Oxford, v. 60, n. 3, p. 849-872, 1972b.

BUCHMAN, R. G. Mortality functions. In: USDA-Forest Service. A generalized forest growth projection system applied to the Lake States regions. Saint Paul: Forest Service/North Central Forest Experiment Station, 1979. p. 47-55. (General Technical Report, n. 49) 
BUCHMAN, R. G.; PEDERSON, S. P.; WALTERS, N. R. A tree survival model with application to species of the Great Lakes region. Canadian Journal of Forest Research, Ottawa, v. 13, n. 4, p. 601608, 1983.

BUGMANN, H. K. M. A simplified forest model to study species composition along climate gradient. Ecology, Copenhagen, v. 77, n. 7, p. 2055-2074, 1996.

CAREY, E. V.; BROWN, S.; GILLESPIE, A. J. R.; LUGO, A. E. Tree mortality in mature lowland tropical moist and tropical lower montane moist forests of Venezuela. Biotropica, Lawrence, v. 26, n. 3, p. 255-265, 1994.

CARVALHO, J. O. P. Dinâmica de florestas naturais e sua implicação para o manejo florestal. In: CURSO DE MANEJO FLORESTAL SUSTENTÁVEL. Tópicos em manejo florestal sustentável. Colombo: Embrapa-CNPF, 1997. p. 43-55.

CASTRO MARÍN, G.; NYGARD, R.; GONZALES RIVAS, B.; ODEN, P. C. Stand dynamics and basal area change in a tropical dry forest reserve in Nicaragua. Forest Ecology and Management, Amsterdam, v. 208, n. 1-3, p. 63-75, 2005.

Van DAALEN, J. C.; SHUGART, H. H. OUTENIQUA - a computer model to simulate succession in the mixed evergreen forests of southern Cape, South Africa. Landscape Ecology, Den Haag, v. 2, n. 4, p. 255$267,1989$.

DEEN, J. L. A survival table for even-aged stands of northern white pine. Journal of Forestry, Washington, v. 31, n. 1, p. 42-44, 1933.

EK, A. R.; MONSERUD, R. A. FOREST: a computer model for simulating the growth and reproduction of mixed species forest stands. Madison: Wisconsin University, 1974. 13 p. (Research report, R2635)

FELFILI, J. M. Structure and dynamics of a gallery forest in central Brazil.. $180 \mathrm{f}$. Thesis (Doctor of Philosophy) - Oxford Forestry Institute/Department of Plant Sciences, University of Oxford, Oxford, 1993.

FINEGAN, B.; CAMACHO, M. Stand dynamics in a logged and silviculturally treated Costa Rican rain forest, 1988-1996. Forest Ecology and Management, Amsterdam, v. 121, n. 3, p. 177-189, 1999.

GERTNER, G. Z.; DZIALOWY, P. J. Effects of measurement errors on an individual tree-based growth projection system. Canadian Journal of Forest Research, Ottawa, v. 14, n. 3, p. 311-316, 1984.

De GRAAF, N. R.; POELS, R. L. H.; Van ROMPAEY, R. S. A. R. Effect of silvicultural treatment on growth and mortality of rainforest in Surinam over long periods. Forest Ecology and Management, Amsterdam, v. 124, n. 2-3, p. 123-135, 1999.

GUAN, B. T.; GERTNER, G. Using a parallel distributed processing system to model individual tree mortality. Forest Science, Bethesda, v. 37, n. 3, p. 871-885, 1991.

HAMILTON Jr., D. A. A logistic model of mortality in thinned and unthinned mixed conifer stands of Northern Idaho. Forest Science, Bethesda, v. 32, n. 4, p. 989-1000, 1986.

HAMILTON Jr., D. A. Extending the range of applicability of an individual tree mortality model. Canadian Journal of Forest Research, Ottawa, v. 20, n. 8, p. 1212-1218, 1990.

HAMILTON Jr., D. A.; EDWARDS, B. M. Modeling the probability of individual tree mortality. Ogden: Forest Service / Intermountain Forest and Range Experiment Station, 1976. 22 p. (Research Paper, n. 185)

HANN, D. W. Development and evaluation of an even- and uneven-aged ponderosa pine/Arizona Fescue stand simulator. Ogden: Forest Service/Intermountain Forest and Range Experiment Station, 1980. 95 p. (Research Paper, n. 267)

HAWKES, C. Woody plant mortality algorithms: description, problems and progress. Ecological Modelling, Amsterdam, v. 126, n. 2-3, p. 225-248, 2000.

KAMMESHEIDT, L.; KÖHLER, P.; HUTH, A. Sustainable timber harvesting in Venezuela: a modeling approach. Journal of Applied Ecology, Oxford, v. 38, n. 4, p. 756-770, 2001. 
KHATOURI, M.; DENNIS, B. Growth-and-yield model for uneven-aged Cedrus atlantica stands in Morocco. Forest Ecology and Management, Amsterdam, v. 36, n. 2-4, p. 253-266, 1990.

KÖHLER, P.; DITZER, T.; ONG, R. C.; HUTH, A. Comparison of measured and modelled growth on permanent plots in Sabahs rain forests. Forest Ecology and Management, Amsterdam, v. 144, n. 1-3, p. 101-111, 2001.

KOHYAMA, T. Simulation of the structural development of warm-temperate rain forest stands. Annals of Botany, London, v. 63, n. 3, p. 625-634, 1989.

KOHYAMA, T. Simulating stationary size distribution of trees in rain forests. Annals of Botany, London, v. 68, p. 173-180, 1991.

KOHYAMA, T. Size-structured multi-species model of rain forest trees. Functional Ecology, Oxford, v. 6, n. 2, p. 206-212, 1992.

KOHYAMA, T. Size-structured tree populations in gap-dynamic forest - the forest architecture hypothesis for the stable coexistence of species. Journal of Ecology, Oxford, v. 81, p. 131-143, 1993.

KOHYAMA, T.; SUZUKI, E.; PARTOMIHARDJO, T.; YAMADA, T. Dynamic steady state of patchmosaic tree-size structure of a mixed dipterocarp forest regulated by local crowding. Ecological Research, Tsukuba, v. 16, n. 1, p. 85-98, 2001.

KUBO, T.; KOHYAMA, T.; POTTS, M. D.; ASTHON, P. S. Mortality rate estimation when inter-census intervals vary. Journal of Tropical Ecology, Cambridge, v. 16, n. 5, p. 753-756, 2000.

LEE, Y. Predicting mortality for even-aged stands of lodgepole pine. Forestry Chronicle, Saint Annede-Bellevue, v. 47, n. 1, p. 29-32, 1971.

LIEBERMAN, D.; LIEBERMAN, M. Forest tree growth and dynamics at La Selva, Costa Rica (19691982). Journal of Tropical Ecology, Cambridge, v. 3, n. 4, p. 347-358, 1987.

LYNCH, T. B.; MOSER Jr., J. W. A growth model for mixed species stands. Bethesda, v. 32, n. 3, p. 697-706, 1986.

MANOKARAN, N.; KOCHUMMEN, K. M. Recruitment, growth and mortality of tree species in a lowland dipterocarp forest in Peninsular Malaysia. Journal of Tropical Ecology, Cambridge, v. 3, n. 4, p. 315-330, 1987.

MONSERUD, R. A. Simulation of forest tree mortality. Forest Science, Bethesda, v. 22, n. 4, p. 438444, 1976.

MOSER Jr., J. W. Dynamics of an uneven-aged forest stand. Forest Science, Bethesda, v. 18, n. 3, p. 184191, 1972.

NAMAALWA, J.; EID, T.; SANKHAYAN, P. A multi-species density-dependent matrix growth model for the dry woodlands of Uganda. Forest Ecology and Management, Amsterdam, v. 213, n. 1-3, p. 312$327,2005$.

OLIVER, C. D.; LARSON, B. C. Forest Stand Dynamics. New York: J. Wiley, 1996. 520 p.

PACALA, S. W.; CANHAM, C. D.; SILANDER Jr., J. A. Forest models defined by field measurements: I. The design of a northeastern forest simulator. Canadian Journal of Forest Research, Ottawa, v. 23, n. 10, p. 1980-1988, 1993.

PHILlIPS, O. L.; HALL, P.; GENTRY, A. H.; SAWYER, S. A.; VÁSQUEZ, R. Dynamics and species richness of tropical rain forests. Proceedings of the National Academy of Sciences of United States of America, Washington, v. 91, n. 7, p. 2805-2809, 1994.

PHILLIPS, P. D.; BRASH, T. E.; YASMAN, I.; SUBAGYO, P.; GARDINGEN, P. R. An individualbased spatially explicit tree growth model for forests in East Kalimantan (Indonesian Borneo). Ecological Modelling, Amsterdam, v. 159, n. 1, p. 1-26, 2003. 
PULZ, F. A. Estudo da dinâmica e a modelagem da estrutura diamétrica de uma floresta semidecídua montana na região de Lavras - MG. 155 f. Dissertação (Mestrado em Engenharia Florestal) - Universidade Federal de Lavras, Lavras, 1998.

REINEKE, L. H. Perfecting a stand-density index for even-aged forests. Journal of Agricultural Research, Washington, v. 46, n. 7, p. 627-638, 1933.

RENNOLLS, K.; PEACE, A. Flow models of mortality and yield for unthinned forest stands. Forestry, London, v. 59, n. 1, p. 47-58, 1986.

ROBERT, A. Simulation of the effect of topography and tree falls on stand dynamics and stand structure tropical forests. Ecological Modelling, Amsterdam, v. 167, n. 3, p. 287-303, 2003.

SANQUETTA, C. R.; CÔRTE, A. P. D.; EISFELD, R. L. Crescimento, mortalidade e recrutamento em duas florestas de Araucária (Araucaria angustifolia (Bert.) O. Ktze.) no estado do Paraná, Brasil. Revista Ciências Exatas e Naturais, Guarapuava, v. 5, n. 1, p. 101-112, 2003.

SHEIL, D. A critique of permanent plot methods and analysis with examples from Budongo Forest, Uganda. Forest Ecology and Management, Amsterdam, v. 77, n. 1-3, p.11-34, 1995.

SHEIL, D.; BURSLEM, D. F. R. P.; ALDER, D. The interpretation and misinterpretation of mortality rate measures. Journal of Ecology, Oxford, v. 83, n. 2, p. 331-333, 1995.

SHEIL, D.; MAY, R. M. Mortality and recruitment rate evaluations in heterogeneous tropical forests. Journal of Ecology, Oxford, v. 84, n. 1, p. 91-100, 1996.

SILVA, J. N. M.; CARVALHO, J. O. P.; LOPES, J. C. A.; ALMEIDA, B. F.; COSTA, D. H. M.; OLIVEIRA, L. C.; VANCLAY, J. K.; SKOVSGAARD, J. P. Growth and yield of a tropical rain forest in the brazilian Amazon after 13 years after logging. Forest Ecology and Management, Amsterdam, v. 71, n. 3, p. 267-274, 1995.

SPATHELF, P.; DURLO, M. A. Transition matrix for modeling the dynamics of a subtropical seminatural forest in southern Brazil. Forest Ecology and Management, Amsterdam, v. 151, n. 1-3, p. 139-149, 2001.

STAGE, A. R.; RENNER, D. L. Comparison of yield-forecasting techniques using long-term stand histories. In: EK, A. R.; SHIFLEY, S. R.; BURK, T. E. (Eds.) Forest Growth and Yield Modeling and Prediction. Proceedings of IUFRO Conference, p. 810-917. 1988. St. Paul: Forest Service/North Central Forest Experiment Station (General Technical Report, n. 120)

SWAINE, M. D.; HALL, J. B.; ALEXANDER, I. J. Tree population dynamics at Kade, Ghana (19681982). Journal of Tropical Ecology, Cambridge, v. 3, n. 4, p. 331-345, 1987.

VANCLAY, J. K. Techniques for modelling timber yield from indigenous forests with special reference to Queensland. 194 f. Dissertation (Master of Science in Forestry) - Linacre College, Oxford University, Oxford, 1983.

VANCLAY, J. K. A growth model for north Queensland rainforests. Forest Ecology and Management, Amsterdam, v. 27, n. 3-4, p. 245-271, 1989.

VANCLAY, J. K. Mortality functions for north Queensland rain forests. Journal of Tropical Forest Science, Kuala, v. 4, n. 1, p. 15-36, 1991.

VANCLAY, J. K. Modelling forest growth and yield. Wallingford: CAB International, 1994a. 312 p.

VANCLAY, J. K. Sustainable timber harvesting: simulation studies in the tropical rainforests of north Queensland. Forest Ecology and Management, Amsterdam, v. 69, n. 1-3, p. 299-320, 1994b.

VANCLAY, J. K. Growth models for tropical forests: a synthesis of models and methods Forest Science, Bethesda, v. 41, n. 1, p. 7-42, 1995.

WEBER, L. A.; EK, A. R.; DROESSLER, T. D. Comparison of stochastic and deterministic mortality estimation in an individual tree based stand growth model. Canadian Journal of Forest Research, Ottawa, v. 16, n. 5, p. 1139-1141, 1986. 
WYCKOFF, P. H.; CLARK, J. S. The relationship between growth and mortality for seven co-occurring tree species in the southern Appalachian Mountains. Journal of Ecology, Oxford, v. 90, n. 4, p. 604-615, 2002.

WYKOFF, W. R. Supplement to the user's guide for the Stand Prognosis Model-version 5.0. Ogden: Forest Service/Intermountain Research Station, 1986. 36 p. (General Technical Report, n. 208)

WYKOFF, W. R.; WIILIAM, R.; CROOKSTON, N. L.; STAGE, A. R. User's Guide to the Stand Prognosis Model. Ogden: Forest Service/Intermountain Forest and Range Experiment Station, 1982. 112 p. (General Technical Report, n. 133)

YANG, Y.; TITUS, S. J. Maximum size-density relationship for constraining individual tree mortality functions. Forest Ecology and Management, Amsterdam, v. 168, n. 1-3, p. 259-273, 2002.

YANG, Y.; TITUS, S. J.; HUANG, S. Modeling individual tree mortality for white spruce in Alberta. Ecological Modeling, Amsterdam, v. 163, n. 3, p. 209-222, 2003.

ZHAO, D.; BORDERS, B.; WILSON, M. Individual-tree diameter growth and mortality models for bottomland mixed-species hardwood stands in the lower Mississippi alluvial valley. Forest Ecology and Management, Amsterdam, v. 199, n. 2-3, p. 307-322, 2004. 